\title{
Phasor model of Modular Multilevel Converter with Circulating Current Suppression Control
}

\author{
Dragan Jovcic, Senior Member,IEEE and Aliakbar Jamshidi Far
}

\begin{abstract}
This paper presents model for Modular Multilevel Converter (MMC) in phasor format, which is convenient for power flow and parameter studies. The model is derived in rotating $d q$ coordinate frame, and the coordinate frame at double the fundamental frequency, in steady-state. A substantial analytical basis is presented in order to facilitate direct mathematical manipulations of non-linear terms in the rotating frame. An $8^{\text {th }}$ order model is firstly derived which includes circulating current representation. Later, the circulating current suppression controller (CCSC) is modelled and the magnitude of second harmonic control inputs is studied. The final model for MMC with CCSC is given in a simple and convenient form for power flow studies. The accuracy of the proposed models is verified against a detailed dynamic MMC benchmark model in PSCAD. Finally, a comparison between the MMC model and 2level VSC model is given.
\end{abstract}

Index Terms-Converter modeling, DC power transmission, MMC, CCSC.

\section{INTRODUCTION}

In recent years, Modular Multilevel Converter (MMC) has become dominant topology for High Voltage Direct Current (HVDC) applications [1]-[3]. Compared to conventional two/three level voltage-source converter (VSC), MMC allows higher power-handling capability with reduced switching power losses and harmonic distortion [4].

The detailed non-linear dynamic models of MMC with different modulation techniques are presented in [5],[6]. These models are developed in PSCAD/EMTDC, which represents detailed characteristics of all switches. These detailed models are discrete in nature and require a considerable amount of simulation time. The model complexity and computation burden is increasing when the number of levels increases.

To overcome these issues with detailed model, the average models are introduced [1],[6] and [7]. The aim of average modeling is to replicate the average response of switching devices, converters and controls by using mathematical equations and controlled voltage or current sources. These MMC average models are very accurate and suitable for transient simulation, but they are implemented in static $a b c$ frame, and are suitable only for time-domain simulation.

Phasor domain models use $d q$ (active and reactive) components of all variables assuming that frequency is constant and all dynamics are neglected. They are required for power flow studies and power system analysis in steady-state, like parametric studies or component dimensioning. The advantage of Phasor modeling is in the simulation speed (elimination of oscillating variables) and parametric steadystate studies ( $a b c$ frame models support only time-domain

This project is funded by European Research Council under the Ideas program in FP7; grant no 259328, 2010.

The authors are with the School of Engineering, University of Aberdeen, Aberdeen, AB24 3UE, U.K. (d.jovcic@abdn.ac.uk, ajamshidifar@abdn.ac.uk). studies). It is generally simple to derive phasor model from dynamic equations for common linear systems, $d q$ frame modeling becomes much more challenging with complex nonlinear systems like MMC converter.

Reference [8] proposes a dynamic phasor modeling for MMC. However, it does not derive steady-state model, the number of equations is high (98 states), and detailed verification is not provided. The analytical MMC model in [9] employs equivalent fixed capacitor on DC side, which is an overly simplified approach under varying operating conditions and second harmonic is neglected altogether. The MMC power flow model is studied in [10], but the model is derived only in $a b c$ frame with oscillating input variables.

Reference [11] presents a detailed harmonic function analysis of the arm currents and the relation between the arm inductance and capacitance at resonant frequencies. Although it is a valuable work regarding higher harmonics, it does not propose full steady-state model for MMC and the circulating current suppression controller (CCSC) is not considered.

Reference [12] derives formulae for all harmonics using a similar approach from [11], and it makes contribution in analysis of elimination of circulating current second harmonic.

In this article, the elementary sinusoidal signals are employed to study their modulation with control signals in time domain. These results will be used to develop analytical theory for representing non-linear multiplying terms in the rotating $d q$ frame. The developed formulae will then be applied to the complex terms of MMC model directly in the rotating frame.

The model will firstly consider in detail all second harmonic terms since they are important for power flow. In the last part, the study considers the case when circulating current suppression controller (CCSC) [13] is active and second harmonic is suppressed. The aim is to produce a sufficiently simple Phasor model for MMC and to verify accuracy.

\section{MMC TIME DOMAIN DYNAMIC MODEL}

Fig.1 shows the structure of one phase leg of MMC [1], [6] and [7]. It consists of two arms (positive and negative) per each phase (x). Each arm comprises of $N$ sub-modules (SMs), one equivalent resistor $R_{\text {armx }}$, and one inductor $L_{a r m x}$ which is required to smooth the voltage difference and phase current.

An average dynamic model for MMC is developed by substituting the arm SMs with an equivalent controlled voltage source as shown in Fig. 1. [1],[6],[7]:

$$
\frac{d}{d t}\left[\begin{array}{c}
I_{\text {diff }} \\
V_{\mathrm{CP}}^{\Sigma} \\
V_{\mathrm{CN}}^{\Sigma}
\end{array}\right]=\left[\begin{array}{ccc}
-\frac{R_{\mathrm{arm}}}{L_{\mathrm{arm}}} & \frac{m_{\mathrm{P}}}{2 L_{\mathrm{arm}}} & \frac{m_{\mathrm{N}}}{2 L_{\text {arm }}} \\
\frac{-m_{\mathrm{P}}}{C^{a r m}} & 0 & 0 \\
\frac{-m_{\mathrm{N}}}{C^{a r m}} & 0 & 0
\end{array}\right]\left[\begin{array}{l}
I_{\text {diff }} \\
V_{\mathrm{CP}}^{\Sigma} \\
V_{\mathrm{CN}}^{\Sigma}
\end{array}\right]+\left[\begin{array}{l}
\frac{-V_{\mathrm{DC}}}{2 L_{\mathrm{arm}}} \\
\frac{-m_{\mathrm{P}} I_{\mathrm{v}}}{2 C^{\mathrm{arm}}} \\
\frac{m_{\mathrm{N}} I_{\mathrm{v}}}{2 C^{\mathrm{arm}}}
\end{array}\right]
$$


where $C^{a r m}=C / N, C$ is the capacitance of one $\mathrm{SM}, N$ is the number of SMs per arm, $I_{\text {diff }}$ is the differential current, $V_{C P}$ and $V_{C N}$ are the positive and negative pole voltages, $V_{\mathrm{CP}}^{\Sigma}$ and $V_{\mathrm{CN}}^{\Sigma}$ are equivalent sum (maximal) voltages of positive and negative arms, $I_{v}$ is the converter ac side current, $V_{D C}$ and $I_{D C}$ are the DC bus voltage and current, and $m_{P}$ and $m_{N}$ are modulation indices of positive and negative arms.

When the average value modeling is used [6],[7], the MMC submodules are replaced with a controllable voltage source as shown in Fig 2, where the converter AC voltage is $e_{x}$ :

$$
e_{x}=\frac{1}{2} m_{N x} V_{C N x}^{\Sigma}-m_{P x} V_{C P_{x}}^{\Sigma}
$$

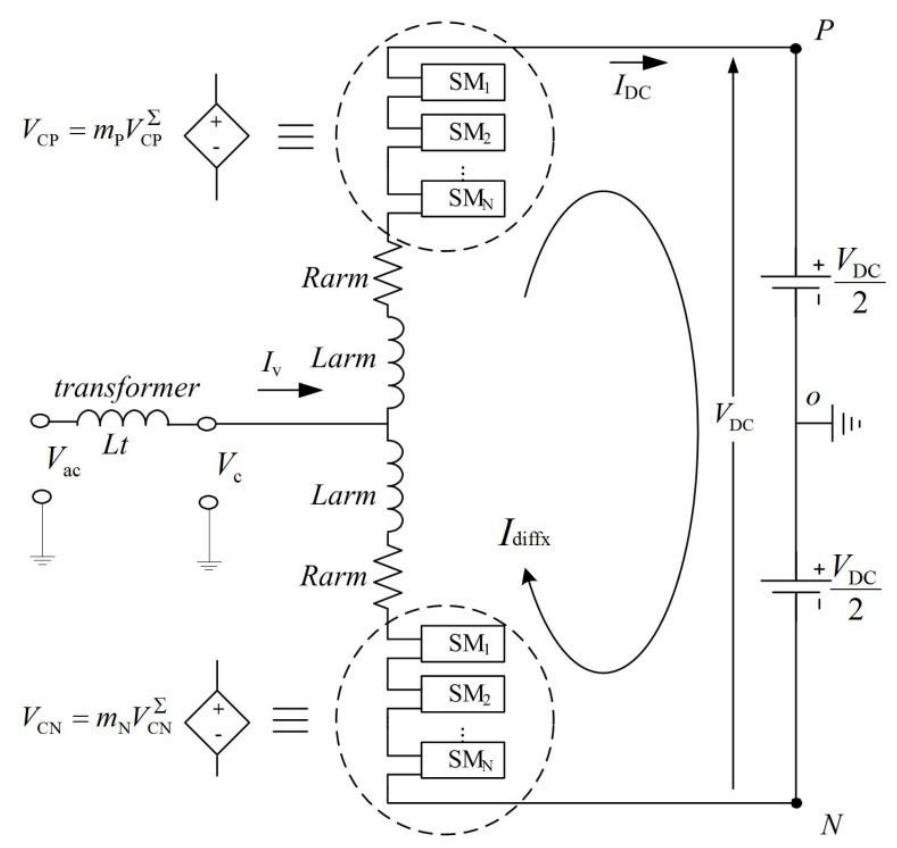

Fig. 1. Circuit diagram of one phase (x) leg of MMC

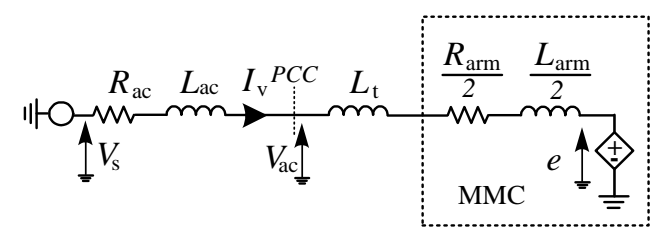

Fig. 2. Structural diagram of the average MMC model.

\section{MMC PHASOR MODEL}

The inputs to the phasor model are assumed to be: $d q$ components of the AC current $I_{v}\left(I_{v d}\right.$ and $\left.I_{v q}\right), d q$ components of the control signal $m\left(M_{d}, M_{q}\right)$ and DC voltage $V_{d c}$. The aim is to derive expressions for dc current $I_{d c}, d q$ components of converter AC voltage $e\left(e_{d}\right.$ and $\left.e_{q}\right), d q$ components of second harmonic circulating current $I_{\text {diff }}\left(I_{\text {diffd } 2}\right.$ and $\left.I_{\text {diffa } 2}\right)$, and also second harmonic arm voltages.

\section{A. Assumptions}

The standard MMC average modeling assumptions are:

- modulation index $m(t)$, is a fundamental sine signal,

- AC current $I_{\mathrm{v}}(t)$, is fundamental sine signal,

- differential current $I_{\text {diff }}(t)$, is DC plus second harmonic,
- sum capacitor voltages $V_{C P}^{\Sigma}(t), V_{C N}^{\Sigma}(t)$, are DC, fundamental and second harmonic, These signals are represented as follows:

$$
\begin{aligned}
& m_{P}=\frac{1}{2} 1-M \cos \left(\omega t-\theta_{m}\right)= \\
& \left(\frac{1}{2}\right)_{0}+\left(-\frac{M_{d}}{2}\right)_{\mathrm{d}} \cos (\omega t)+\left(-\frac{M_{q}}{2}\right)_{\mathrm{q}} \sin (\omega t) \\
& m_{N}=\frac{1}{2} 1+M \cos \left(\omega t-\theta_{m}\right)= \\
& \left(\frac{1}{2}\right)_{0}+\left(\frac{M_{d}}{2}\right)_{\mathrm{d}} \cos (\omega t)+\left(\frac{M_{q}}{2}\right)_{\mathrm{q}} \sin (\omega t) \\
& I_{V}(t)=I_{V m} \cos \left(\omega t-\theta_{i}\right)=I_{V_{d}}+I_{V} q_{q} \\
& I_{\text {diff }}(t)=I_{\text {diff } 0}+I_{\text {diff } 2} \cos \left(2 \omega t-\theta_{\text {idiff } 2}\right)=I_{\text {diff } 0}+I_{\text {diff }} d_{22}+I_{\text {diff }} q^{2} \\
& V_{C P}(t)=V_{C P 0}+V_{C P m} \cos \left(\omega t+\theta_{V P}\right)+V_{C P m 2} \cos \left(2 \omega t+\theta_{V P 2}\right) \\
& =V_{C P 0}+V_{C P d d}+V_{C P q_{q}}+V_{C P d 2 d 2}+V_{C P q 2}{ }_{q 2} \\
& V_{C N}(t)=V_{C N 0}+V_{C N m} \cos \left(\omega t+\theta_{V N}\right)+V_{C N m 2} \cos \left(2 \omega t+\theta_{V N 2}\right)(7) \\
& =V_{C N 0}+V_{C N d d}+V_{C N q_{q}}+V_{C N d 2_{d 2}}+V_{C N q 2} q 2
\end{aligned}
$$

Where $d, q$ subscripts denote the two components in the coordinate frame rotating at fundamental frequency $\omega=2 \pi f$, while $d 2, q 2$ subscripts denote the two components in the coordinate frame rotating at second harmonic $2 \omega=4 \pi f$, and subscript 0 denotes zero sequence component.

\section{B. Zero sequence model}

Model (1) can be rewritten for zero sequence as follows:

$$
\begin{aligned}
& 0=-R_{\text {arm }} I_{\text {diff } 0}+\left(\frac{m_{P} V_{C P}}{2}\right)_{0}+\left(\frac{m_{N} V_{C N}}{2}\right)_{0}-\frac{V_{D C}}{2} \\
& 0=-m_{P} I_{\text {diff }} 0-\left(\frac{m_{P}}{2} I_{V}\right)_{0} \\
& 0=-m_{N} I_{\text {diff }_{0}}+\left(\frac{m_{N}}{2} I_{V}\right)_{0}
\end{aligned}
$$

In (8), it is required to derive zero sequence of the product of two phasor signals. Appendix I gives generic expression for multiplication of two phasors which may contain zero sequence, fundamental frequency and second harmonics, in $d q$ frame. Therefore, using (43), the last equation of model (8) gives DC component of differential current:

$$
I_{d i f f 0}=M_{d} I_{v d}+M_{q} I_{v q} / 4
$$

The DC current is the sum of zero sequence currents in the three phases which gives the DC side equation known from average modeling [7]:

$$
I_{d c}=3 I_{d i f f 0}=3 \quad M_{d} I_{v d}+M_{q} I_{v q} / 4
$$

\section{Fundamental frequency model}

The fundamental frequency model needs to be derived separately for positive and negative poles. The second equation of (1) takes the following form in $d q$ frame: 


$$
\begin{aligned}
& C^{a r m}\left[\begin{array}{lll}
-\omega V_{C P q_{d}}^{\Sigma}+\omega V_{C P d}^{\Sigma} &
\end{array}\right]= \\
& -m_{P} I_{\text {diff }_{d}}-m_{P} I_{V} / 2_{d}-m_{P} I_{\text {diff }}-m_{P} I_{V} / 2_{q}
\end{aligned}
$$

The corresponding $d$ and $q$ components of the product terms can be determined form the algebra (43) in the Appendix I:

$$
\begin{aligned}
& V_{C P d}^{\Sigma}=\frac{1}{\omega C^{a r m}}\left(\frac{M_{q}}{2} I_{d i f f 0}-\frac{M_{q}}{4} I_{d i f f d 2}+\frac{M_{d}}{4} I_{d i f f{ }^{2}}-\frac{I_{V q}}{4}\right) \\
& V_{C P q}^{\Sigma}=\frac{1}{\omega C^{a r m}}\left(-\frac{M_{d}}{2} I_{d i f f 0}-\frac{M_{d}}{4} I_{d i f f d 2}-\frac{M_{q}}{4} I_{d i f f q^{2}}+\frac{I_{V d}}{4}\right)
\end{aligned}
$$

It is observed that in the above expression the variables from zero sequence model $\left(I_{\text {diffo }}\right)$ and also from the second harmonic model $\left(I_{\text {diffd } 2}, I_{\text {diffq } 2}\right)$ are present, which denotes crosscoupling between the three coordinate frames.

By expanding the first equation of (8) and also assuming $m_{P} V_{C P}^{\Sigma}{ }_{0}=m_{N} V_{C N}^{\Sigma}$, the DC component of $V_{C P}^{\Sigma}$ is:

$$
\begin{aligned}
& V_{C P 0}^{\Sigma}=2 R_{\text {arm }} I_{\text {diff } 0}+V_{D C} \\
& -\frac{1}{\omega C^{\text {arm }}}\left(\frac{M_{d} I_{V q}}{8}-\frac{M_{q} I_{V d}}{8}+\frac{M_{d} M_{q}}{4} I_{\text {diff } 2}+\frac{-M_{d}^{2}+M_{q}^{2}}{8} I_{\text {diff } q 2}\right)
\end{aligned}
$$

The equations for the negative pole sum voltages can be developed in a similar way starting from third equation in (1):

$$
\begin{aligned}
& V_{C N d}^{\Sigma}=\frac{1}{\omega C^{a r m}}\left(-\frac{M_{q}}{2} I_{\text {diff } 0}+\frac{M_{q}}{4} I_{\text {diffd } 2}-\frac{M_{d}}{4} I_{d i f f q 2}+\frac{I_{V q}}{4}\right) \\
& V_{C N q}^{\Sigma}=\frac{1}{\omega C^{a r m}}\left(\frac{M_{d}}{2} I_{\text {diff } 0}+\frac{M_{d}}{4} I_{\text {diffd } 2}+\frac{M_{q}}{4} I_{d i f f q 2}-\frac{I_{V d}}{4}\right) \\
& V_{C N 0}^{\Sigma}=2 R_{\text {arm }} I_{\text {diff } 0}+V_{D C} \\
& -\frac{1}{\omega C^{a r m}}\left(\frac{M_{d} I_{V q}}{8}-\frac{M_{q} I_{V d}}{8}+\frac{M_{d} M_{q}}{4} I_{\text {diffd } 2}+\frac{-M_{d}^{2}+M_{q}^{2}}{4} I_{\text {diffd } 2}\right)
\end{aligned}
$$

\section{Second harmonic model}

Starting from the second and third equations in model (1), and considering the expression for second harmonic of a product of two signals from (43) in Appendix I, the $d 2, q 2$ components for $V_{\mathrm{CP}}^{\Sigma}, V_{\mathrm{CN}}^{\Sigma}$ are calculated as:

$$
\begin{aligned}
& V_{C P d 2}^{\Sigma}=\frac{1}{2 \omega C^{a r m}}\left[-\frac{1}{2} I_{d i f f q 2}+\frac{M_{q} I_{V d}}{8}+\frac{M_{d} I_{V q}}{8}\right] \\
& V_{C P q 2}^{\Sigma}=\frac{1}{2 \omega C^{a r m}}\left[\frac{1}{2} I_{d i f f d 2}-\frac{M_{d} I_{V d}}{8}+\frac{M_{q} I_{V q}}{8}\right]
\end{aligned}
$$

And similarly for negative pole:

$$
\begin{aligned}
& V_{C N d 2}^{\Sigma}=\frac{1}{2 \omega C^{a r m}}\left[-\frac{1}{2} I_{d i f f q 2}+\frac{M_{q} I_{V d}}{8}+\frac{M_{d} I_{V q}}{8}\right] \\
& V_{C N q 2}^{\Sigma}=\frac{1}{2 \omega C^{\text {arm }}}\left[\frac{1}{2} I_{\text {diff } 22}-\frac{M_{d} I_{V d}}{8}+\frac{M_{q} I_{V q}}{8}\right]
\end{aligned}
$$

It is clear from (16) and(17) that second harmonic variables depend on the fundamental components, i.e. on converter loading. By comparing (13) with (15), (12) with (14), and (16) with (17), the following equalities are observed:

$$
\begin{aligned}
& V_{C N 0}^{\Sigma}=V_{C P 0}^{\Sigma} \\
& V_{C P d}^{\Sigma}=-V_{C N d}^{\Sigma}, \quad V_{C P q}^{\Sigma}=-V_{C N q}^{\Sigma} \\
& V_{C P d 2}^{\Sigma}=V_{C N d 2}^{\Sigma}, \quad V_{C P q 2}^{\Sigma}=V_{C N q 2}^{\Sigma}
\end{aligned}
$$

The circulating current (first) equation in model (1) can also be written for second harmonic as:

$$
\begin{aligned}
& -2 \omega L_{\text {arm }} I_{d i f f 2_{d 2}}+2 \omega L_{\text {arm }} I_{d i f f d 2} q_{q^{2}}=-R_{\text {arm }} I_{\text {diffd }{ }_{d 2}}+-R_{\text {arm }} I_{d i f f q^{2}} \\
& +\left(\frac{m_{P x} V_{C P x}}{2}+\frac{m_{N x} V_{C N x}}{2}\right)_{d 2}+\left(\frac{m_{P x} V_{C P x}}{2}+\frac{m_{N x} V_{C N x}}{2}\right)_{q 2}
\end{aligned}
$$

By separating (19) into $d 2$ and $q 2$ components:

$$
\begin{aligned}
I_{\text {diff } 2} & =\frac{1}{2 \omega L_{\text {arm }}}\left(-R_{\text {arm }} I_{\text {diff } q 2}-\frac{M_{d} V_{C P q}^{\Sigma}}{4}-\frac{M_{q} V_{C P d}^{\Sigma}}{4}+\frac{V_{C P q 2}^{\Sigma}}{2}\right)(20) \\
I_{\text {diff } 2} & =\frac{1}{2 \omega L_{\text {arm }}}\left(R_{\text {arm }} I_{\text {diff } 22}+\frac{M_{d} V_{C P d}^{\Sigma}}{4}-\frac{M_{q} V_{C P q}^{\Sigma}}{4}-\frac{V_{C P d 2}^{\Sigma}}{2}\right)
\end{aligned}
$$

Eq. (20) can be used to derive the magnitude of circulating current, $I_{\text {diff } 2}$, in terms of AC currents and modulation indices. The derivation is given in Appendix II.

\section{E. The expression for MMC AC voltage}

The $d q$ components of the MMC AC voltage in (2) are:

$$
\begin{aligned}
& e_{d}=\frac{1}{2} m_{N x} V_{C N x_{d}}^{\Sigma}-m_{P x} V_{C P x_{d}}^{\Sigma} \\
& e_{q}=\frac{1}{2} m_{N x} V_{C N x_{q}}^{\Sigma}-m_{P x} V_{C P x_{q}}^{\Sigma}{ }_{q}
\end{aligned}
$$

By expanding the multiplication terms of (21):

$$
\begin{aligned}
& e_{d}=-2 V_{C P d}^{\Sigma}+2 M_{d} V_{C P 0}^{\Sigma}+M_{d} V_{C P d 2}^{\Sigma}+M_{q} V_{C P q 2}^{\Sigma} / 4 \\
& e_{q}=-2 V_{C P q}^{\Sigma}+2 M_{q} V_{C P 0}^{\Sigma}-M_{q} V_{C P d 2}^{\Sigma}+M_{d} V_{C P q 2}^{\Sigma} / 4
\end{aligned}
$$

\section{F. Full MMC phasor model in matrix form}

Considering the derivations all three coordinate frames, the phasor model of MMC can be summarized in matrix form as:

$$
A x=B u, \quad y=C x
$$

Where $x, u$ and $y$ are state, input and output vectors:

$$
\begin{aligned}
& x^{T}=\left[\begin{array}{lllll|lll}
V_{C P d 2}^{\Sigma} & V_{C P q 2}^{\Sigma} & V_{C P d}^{\Sigma} & V_{C P q}^{\Sigma} & V_{C P 0}^{\Sigma} & I_{\text {diff } 0} & I_{\text {diffd } 2} & I_{\text {diff } q 2}
\end{array}\right] \\
& u^{T}=\left[\begin{array}{lll}
I_{V d} & I_{V q} & V_{D C}
\end{array}\right], \quad y^{T}=\left[\begin{array}{lll}
e_{d} & e_{q} & I_{D C}
\end{array}\right]
\end{aligned}
$$

The matrices are:

$$
\begin{aligned}
B^{T} & =\frac{1}{16 \omega C^{a r m}}\left[\begin{array}{ccccccccc}
0 & 0 & 0 & 0 & 16 \omega C^{\text {arm }} & 0 & 0 & 0 \\
M_{d} & M_{q} & -4 & 0 & -2 M_{d} & 4 M_{q} \omega C^{a r m} & 0 & 0 \\
M_{q} & -M_{d} & 0 & 4 & 2 M_{q} & 4 M_{d} \omega C^{a r m} & 0 & 0
\end{array}\right] \\
C & =\frac{1}{4}\left[\begin{array}{cccccccc}
M_{d} & M_{q} & -2 & 0 & 2 M_{d} & 0 & 0 & 0 \\
-M_{q} & M_{d} & 0 & -2 & 2 M_{q} & 0 & 0 & 0 \\
0 & 0 & 0 & 0 & 0 & 12 & 0 & 0
\end{array}\right]
\end{aligned}
$$




$$
\begin{aligned}
& A=\left[\begin{array}{ll}
A_{11} & A_{12} \\
A_{21} & A_{22}
\end{array}\right], \quad A_{11}=I_{5 \times 5}, \quad A_{22}=\left[\begin{array}{ccc}
1 & 0 & 0 \\
0 & 1 & \frac{R_{a r m}}{2 \omega L_{a r m}} \\
0 & \frac{-R_{a r m}}{2 \omega L_{a r m}} & 1
\end{array}\right]
\end{aligned}
$$

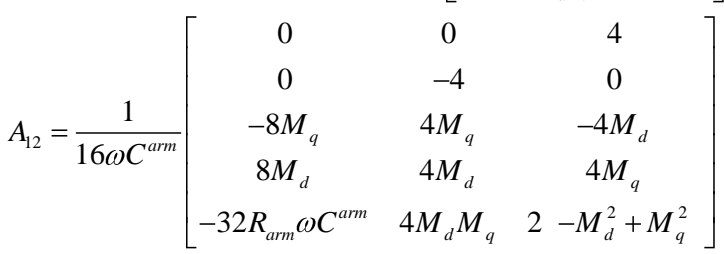

$$
\begin{aligned}
& A_{21}=\frac{1}{8 \omega L_{a r m}}\left[\begin{array}{ccccc}
0 & 0 & 0 & 0 & 0 \\
0 & -2 & M_{q} & -M_{d} & 0 \\
2 & 0 & -M_{d} & M_{q} & 0
\end{array}\right]
\end{aligned}
$$

\section{Circulating Current Control Modelling}

The circulating current is usually eliminated by using feedback PI control [14], in $d 2, q 2$ rotating frame. As a result, the modulation indices will include second harmonic terms $M_{d 2}$ and $M_{q 2}$. Therefore, the following form for the positive and negative modulation indices is considered:

$$
\begin{aligned}
& m_{P}=\frac{1}{2} 1-M \cos \left(\omega t-\theta_{m}\right)-M_{2} \cos \left(2 \omega t-\theta_{m 2}\right)= \\
& \left(\frac{1}{2}\right)_{0}+\left(-\frac{M_{d}}{2}\right)_{\mathrm{d}} \cos (\omega t)+\left(-\frac{M_{q}}{2}\right)_{\mathrm{q}} \sin (\omega t) \\
& +\left(-\frac{M_{d 2}}{2}\right)_{\mathrm{d} 2} \cos (2 \omega t)+\left(-\frac{M_{q 2}}{2}\right)_{\mathrm{q} 2} \sin (2 \omega t) \\
& m_{N}=\frac{1}{2} 1+M \cos \left(\omega t-\theta_{m}\right)-M_{2} \cos \left(2 \omega t-\theta_{m 2}\right)= \\
& \left(\frac{1}{2}\right)_{0}+\left(\frac{M_{d}}{2}\right)_{\mathrm{d}} \cos (\omega t)+\left(\frac{M_{q}}{2}\right)_{\mathrm{q}} \sin (\omega t) \\
& +\left(-\frac{M_{d 2}}{2}\right)_{\mathrm{d} 2} \cos (2 \omega t)+\left(-\frac{M_{q 2}}{2}\right)_{\mathrm{q} 2} \sin (2 \omega t)
\end{aligned}
$$

The second harmonic control terms will modify all equations for the MMC phasor model derived in Section III. It will be assumed that CCSC achieves ideally the control goals: $I_{\text {diffd } 2}=I_{\text {diff } q 2}=0$. Following full derivation, it can be verified that conclusions (18) are still valid. Therefore, the $d q$ components of positive and negative pole voltages are:

$$
\begin{aligned}
& V_{C P d}^{\Sigma}=-V_{C N d}^{\Sigma}=\frac{1}{\omega C^{a r m}}\left(\frac{M_{q}}{2} I_{d i f f 0}-\frac{I_{V q}}{4}-\frac{M_{d 2}}{8} I_{v q}+\frac{M_{q 2}}{8} I_{v d}\right) \\
& V_{C P q}^{\Sigma}=-V_{C N q}^{\Sigma}=\frac{1}{\omega C^{a r m}}\left(-\frac{M_{d}}{2} I_{d i f f 0}+\frac{I_{V d}}{4}-\frac{M_{d 2}}{8} I_{v d}-\frac{M_{q 2}}{8} I_{v q}\right)
\end{aligned}
$$

The zero sequence pole voltages are

$$
\begin{aligned}
& V_{C P 0}^{\Sigma}=V_{C N 0}^{\Sigma}=2 R_{a r m} I_{d i f 0}+V_{D C}+\frac{1}{\omega C^{a r m}}\left(\frac{M_{d} I_{V q}}{8}-\frac{M_{q} I_{V d}}{8}\right) \\
& +\frac{1}{\omega C^{a r m}}\left(\frac{-M_{d} M_{q 2}+M_{q} M_{d 2}}{32} I_{v d}+\frac{M_{d} M_{d 2}+M_{q} M_{q 2}}{32} I_{v q}\right)
\end{aligned}
$$

The second harmonic $d q$ components are derived similarly as in section III.C as follows:

$$
\begin{aligned}
& V_{C P d 2}^{\Sigma}=V_{C N d 2}^{\Sigma}=\frac{1}{2 \omega C^{a r m}}\left(\frac{M_{q 2} I_{d i f f 0}}{2}+\frac{M_{q} I_{V d}}{8}+\frac{M_{d} I_{V q}}{8}\right) \\
& V_{C P q 2}^{\Sigma}=V_{C N q 2}^{\Sigma}=\frac{1}{2 \omega C^{a r m}}\left(-\frac{M_{d 2} I_{d i f f 0}}{2}-\frac{M_{d} I_{V d}}{8}+\frac{M_{q} I_{V q}}{8}\right)
\end{aligned}
$$

The MMC AC voltage is derived by expanding the multiplication terms of (21):

$$
\begin{aligned}
& e_{d}=-2 V_{C P d}^{\Sigma}+2 M_{d} V_{C P 0}^{\Sigma}+M_{d} V_{C P d 2}^{\Sigma}+M_{q} V_{C P q^{2}}^{\Sigma}+M_{d 2} V_{C P d}^{\Sigma}+M_{q 2} V_{C P q}^{\Sigma} / 4 \\
& e_{q}=-2 V_{C P q}^{\Sigma}+2 M_{q} V_{C P 0}^{\Sigma}-M_{q} V_{C P d 2}^{\Sigma}+M_{d} V_{C P{ }^{2} 2}^{\Sigma}+M_{q 2} V_{C P d}^{\Sigma}-M_{d 2} V_{C P q}^{\Sigma} / 4
\end{aligned}
$$

The third model output, $I_{D C}$, is the same as given in (9).

It is now possible to derive the magnitude of control signals $M_{d 2}$ and $M_{q 2}$, required to eliminate second harmonic current. Such result will give theoretical background for possible open loop CCSC. Replacing $I_{d i f f d 2}=I_{d i f f q_{2}}=0$ in (19):

$$
\begin{aligned}
& m_{P x} V_{C P x}+m_{N x} V_{C N x} d 2=0 \\
& m_{P x} V_{C P x}+m_{N x} V_{C N x} \quad=0
\end{aligned}
$$

By expanding (33):

$$
\begin{aligned}
& M_{d 2}=\left(-\frac{M_{d} V_{C P d}^{\Sigma}}{2}+\frac{M_{q} V_{C P q}^{\Sigma}}{2}+V_{C P d 2}^{\Sigma}\right) / V_{C P 0}^{\Sigma} \\
& M_{q 2}=\left(-\frac{M_{q} V_{C P d}^{\Sigma}}{2}-\frac{M_{d} V_{C P q}^{\Sigma}}{2}+V_{C P q 2}^{\Sigma}\right) / V_{C P 0}^{\Sigma}
\end{aligned}
$$

Assuming that the values for $M_{d 2}$ and $M_{q 2}$ are very small, as it will be confirmed in Section VI, the last two terms in (30) are neglected to simplify the derivation:

$$
V_{C P 0}^{\Sigma}=V_{C N 0}^{\Sigma} \approx 2 R_{a r m} I_{d i f f 0}+V_{D C}+\frac{1}{\omega C^{a r m}}\left(\frac{M_{d} I_{V q}}{8}-\frac{M_{q} I_{V d}}{8}\right)(35)
$$

$M_{d 2}$ and $M_{q 2}$ is obtained by replacing (29),(31), (35) in (34):

$$
\begin{aligned}
& {\left[\begin{array}{l}
M_{d 2} \\
M_{q 2}
\end{array}\right]=\frac{1}{Z}\left[\begin{array}{cccc}
3-2 M_{d}^{2} & M_{q} & 3-2 M_{q}^{2} & M_{d} \\
M_{d}^{2}-M_{q}^{2}-3 & M_{d} & M_{d}^{2}-M_{q}^{2}+3 & M_{q}
\end{array}\right]\left[\begin{array}{c}
I_{V d} \\
I_{V q}
\end{array}\right]} \\
& \text { where } \\
& Z=8 \omega C^{a r m} 2 V_{D C}-R_{a r m} \quad M_{d} I_{V d}+M_{q} I_{V q}-3 M_{d} I_{V q}-M_{q} I_{V d}
\end{aligned}
$$

\section{SIMPLIFIED MODEL OF MMC WITH CCSC}

The system model with CCSC is given in (29)-(32). However simulation studies have demonstrated, as it will be shown in verification section, that the magnitude of CCSC control signals $M_{d 2}$ and $M_{q 2}$ even at full power is very small. Therefore it is justified to simplify further the practical MMC model by neglecting CCSC control signals. Therefore, from (29)-(32) the simple MMC phasor model with CCSC is:

$$
\begin{aligned}
& {\left[\begin{array}{l}
e_{d} \\
e_{q}
\end{array}\right]=\left[\begin{array}{ll}
A_{11} & A_{12} \\
A_{21} & A_{22}
\end{array}\right]\left[\begin{array}{l}
I_{V d} \\
I_{V q}
\end{array}\right]+0.5\left[\begin{array}{l}
M_{d} \\
M_{q}
\end{array}\right] V_{D C}} \\
& A_{11}=\frac{M_{d}^{2} R_{a r m}}{4}, A_{12}=-\frac{8-3 M_{d}^{2}+M_{q}^{2}}{64 \omega C^{a r m}}+\frac{M_{d} M_{q} R_{a r m}}{4} \\
& A_{21}=\frac{8-3 M_{d}^{2}+M_{q}^{2}}{64 \omega C^{a r m}}+\frac{M_{q} M_{d} R_{a r m}}{4}, A_{22}=\frac{M_{q}^{2} R_{a r m}}{4}
\end{aligned}
$$


Note that the DC current equation (9) should be included to complete the MMC model in (37). The above MMC steadystate model is in simple convenient form for system dimensioning and power flow studies, while it is very accurate as it will be verified in the next section.

\section{MODEL VERIFICATION}

\section{A. PSCAD benchmark model}

The PSCAD benchmark model consists of a MMC converter represented as given in [5], which is "type 4" from [7], and which is connected to an AC and a simple DC source. The MMC is a 401-level $1000 M V A$ converter with $C_{S M}=10 m F$ and $R_{\text {arm }}=1.2 \Omega$, with $L_{\text {arm }}=0.15 H$ in case without CCSC. In the case the CCSC is active the inductance is $L_{\text {arm }}=0.08 H$. The $\mathrm{AC}$ grid and DC bus voltages are $V_{A C}=370 \mathrm{KV}, V_{D C}=640 \mathrm{KV}$, and AC parameters are: $S C R=8.5, X / R=10, X_{t}=8 \%$.

The model inputs are $M_{d}, M_{q}, I_{v d}, I_{v q}$ and $V_{d c}$, and they have the same values for the PSCAD and the analytical model in all tests. The outputs $e_{d}, e_{q}, I_{\text {diff } 2}$ and $I_{\text {diffo }}$ (also $M_{d 2}, M_{q 2}$ in CCSC tests) are observed and compared with PSCAD Benchmark.

\section{B. Verification of model without CCSC}

The proposed phasor model without CCSC, eq. (23), is verified against detailed PSCAD model for different range of power levels and the results are shown in table I. These power levels are obtained by keeping $M_{d}=0.92$ and manipulating $M_{q}$. The two models are compared for $I_{d i f f o}, e_{d}, e_{q}$ and also $I_{d i f f 2}$ as the outputs. The results confirm excellent matching between the model and detailed PSCAD benchmark.

The phasor model is tested for different $L_{a r m}$ parameters, and for wide range of power levels. For brevity, the results are reported only for full power and three different values of $L_{\text {arm }}$ as shown in Table II. Excellent matching is observed.

TABLE I. VERIFICATION OF MODEL WITHOUT CCSC $\left(\mathrm{L}_{\text {ARM }}=0.15 \mathrm{H}\right)$

\begin{tabular}{|c|c|c|c|c|c|c|c|}
\hline \multicolumn{2}{|c|}{$\mathrm{L}_{\text {arm }}=0.15 \mathrm{H}$} & $\mathrm{P}=0.1 \mathrm{pu}$ & $\mathrm{P}=0.5 \mathrm{pu}$ & $\mathrm{P}=1 \mathrm{pu}$ & $\mathrm{P}=-0.1 \mathrm{pu}$ & $\mathrm{P}=-0.5 \mathrm{pu}$ & $\mathrm{P}=-1 \mathrm{pu}$ \\
\hline$I_{\text {difo }}$ & PSCAD & 0.518 & 0.259 & 0.052 & -0.052 & -0.261 & -0.524 \\
\cline { 2 - 8 }$(K A)$ & Model & 0.518 & 0.259 & 0.052 & -0.052 & -0.261 & -0.524 \\
\hline$I_{\text {diff }}$ & PSCAD & 0.1874 & 0.0924 & 0.0291 & 0.0319 & 0.0955 & 0.186 \\
\cline { 2 - 8 }$(K A)$ & Model & 0.1776 & 0.0878 & 0.0278 & 0.0304 & 0.0907 & 0.176 \\
\hline \multirow{2}{*}{$e_{d}(K V)$} & PSCAD & 208.5 & 207.2 & 206.3 & 205.9 & 205.4 & 204.9 \\
\cline { 2 - 8 } & Model & 208.5 & 207.1 & 206.3 & 205.9 & 205.3 & 204.8 \\
\hline \multirow{2}{*}{$e_{q}(K V)$} & PSCAD & -52.5 & -26.1 & -5.10 & 5.31 & 26.10 & 52.2 \\
\cline { 2 - 8 } & Model & -52.2 & -25.9 & -5.10 & 5.29 & 25.98 & 51.9 \\
\hline
\end{tabular}

TABLE II. VERIFICATION OF MODEL WITHOUT CCSC ( $\mathrm{P}=1 \mathrm{PU})$

\begin{tabular}{|c|c|c|c|c|}
\hline \multicolumn{2}{|c|}{$\mathrm{P}=1 \mathrm{pu}$} & $\mathrm{L}_{\mathrm{arm}}=0.1 \mathrm{H}$ & $\mathrm{L}_{\mathrm{arm}}=0.15 \mathrm{H}$ & $\mathrm{L}_{\mathrm{arm}}=0.2 \mathrm{H}$ \\
\hline$I_{\text {diffo }}(K A)$ & PSCAD & 0.517 & 0.518 & 0.518 \\
\cline { 2 - 5 } & Model & 0.517 & 0.518 & 0.518 \\
\hline \multirow{2}{*}{$I_{\text {diff } 2}(K A)$} & PSCAD & 0.342 & 0.1874 & 0.130 \\
\cline { 2 - 5 } & Model & 0.313 & 0.1776 & 0.1253 \\
\hline \multirow{2}{*}{$e_{d}(K V)$} & PSCAD & 208.6 & 208.5 & 208.5 \\
\cline { 2 - 5 } & Model & 208.6 & 208.5 & 208.5 \\
\hline \multirow{2}{*}{$e_{q}(K V)$} & PSCAD & -39.95 & -52.5 & -65.0 \\
\cline { 2 - 5 } & Model & -39.22 & -52.18 & -64.8 \\
\hline
\end{tabular}

C. Investigation of resonance condition for $L_{\text {arm }}$ and $C^{\text {arm }}$

Fig. 3 shows the magnitude of $I_{\text {diff } 2}$ versus $L_{\text {arm }}$ for 3 values of $C^{\text {arm }}$, using the model (23). The control signals are kept constant: $M_{d}=0.92, M_{d}=-0.055$. Therefore, the power flow is different for every $L_{\text {arm }}$ and $C^{\text {arm }}$. It is equal to $1 p u$ for the following pairs $\left(C^{a r m}=20 u F, \quad L_{\text {arm }}=0.076 H\right), \quad\left(C^{a r m}=25 u F\right.$,
$\left.L_{\text {arm }}=0.059 H\right)$ and $\left(C^{a r m}=30 u F, L_{\text {arm }}=0.047 H\right)$. It is seen that $I_{\text {diff } 2}$ has multiple peaks for each $C^{\text {arm }}$ which correspond to various harmonic resonances. The magnitudes of second harmonic at resonant conditions are very high even with realistic arm resistances and clearly resonances should be avoided.

Following the derivation from Appendix II, the analytical expression for the arm inductance at resonance is given as:

$$
L_{\text {arm_res }}=\frac{2+M_{d}^{2}+M_{q}^{2}}{32 \omega^{2} C^{a r m}}
$$

The values obtained from (38) correspond well with peaks in Fig 3, and the values are close to the resonant inductor values reported in [11] (there is $10 \%$ difference comparing (38) with formula (119) in [11]).

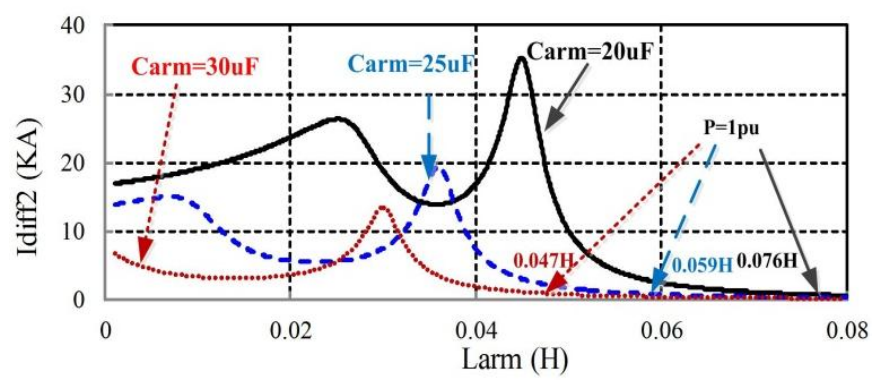

Fig. 3. Idiff2 versus Larm for 3 Carm

\section{Verification of CCSC control mangnitude}

Table III compares the $M_{d 2}$ and $M_{q 2}$ of eq. (36) against the PSCAD model with CCSC for different operating points. The results show good matching for the wide range of power flow. This implies that (36) can be used for open loop active second harmonic suppression which would avoid control dynamic interactions. Also, this table confirms that magnitude of $M_{d 2}$ and $M_{q 2}$ is small, and justifies simplifications in model (37).

TABLE III. VERIFICATION OF CCSC CONTROL MAGNITUDES

\begin{tabular}{|c|c|c|c|c|}
\hline \multirow{2}{*}{$\mathrm{L}_{\text {arm }}=0.08 \mathrm{H}$} & \multicolumn{2}{|c|}{$M_{d 2}$} & \multicolumn{2}{c|}{$M_{q 2}$} \\
\cline { 2 - 5 } & PSCAD & Model & PSCAD & Model \\
\hline $\mathrm{P}=1 \mathrm{pu}$ & -0.0028 & -0.003 & -0.0565 & -0.0557 \\
\hline $\mathrm{P}=0.5 \mathrm{pu}$ & -0.0064 & -0.0064 & -0.0276 & -0.0273 \\
\hline $\mathrm{P}=0.1 \mathrm{pu}$ & -0.0091 & -0.0091 & -0.0055 & -0.0054 \\
\hline $\mathrm{P}=-0.1 \mathrm{pu}$ & -0.0105 & -0.0104 & 0.0546 & 0.0557 \\
\hline $\mathrm{P}=-0.5 \mathrm{pu}$ & -0.013 & -0.0132 & 0.0271 & 0.0267 \\
\hline $\mathrm{P}=-1 \mathrm{pu}$ & -0.016 & -0.0167 & 0.0546 & 0.0536 \\
\hline
\end{tabular}

\section{E. Verification of simplified model with CCSC}

The simplified model with CCSC, eq. (37), is verified against PSCAD MMC benchmark with CCSC active and the results for various power flows are given in table IV. The results show excellent model accuracy despite simplifications.

TABLE IV. VERIFICATION OF SIMPLIFIED MMC MODEL WITH CCSC

\begin{tabular}{|c|c|c|c|c|c|c|c|}
\hline \multicolumn{2}{|c|}{$\mathrm{L}_{\text {arm }}=0.08 \mathrm{H}$} & $\mathrm{P}=0.1 \mathrm{pu}$ & $\mathrm{P}=0.5 \mathrm{pu}$ & $\mathrm{P}=1 \mathrm{pu}$ & $\mathrm{P}=-0.1 \mathrm{pu}$ & $\mathrm{P}=-0.5 \mathrm{pu}$ & $\mathrm{P}=-1 \mathrm{pu}$ \\
\hline$I_{\text {difo }}$ & PSCAD & 0.518 & 0.260 & 0.0519 & -0.052 & -0.261 & -0.524 \\
\cline { 2 - 8 }$(K A)$ & Model & 0.518 & 0.260 & 0.0519 & -0.052 & -0.261 & -0.524 \\
\hline \multirow{2}{*}{$\left.e_{d}(K V)\right)$} & PSCAD & 208.5 & 207.1 & 206.2 & 205.8 & 205.2 & 204.7 \\
\cline { 2 - 8 } & Model & 208.5 & 207.1 & 206.2 & 205.8 & 205.2 & 204.7 \\
\hline \multirow{2}{*}{$e_{q}(K V)$} & PSCAD & -35.00 & -17.40 & -3.38 & 3.62 & 17.60 & 35.05 \\
\cline { 2 - 8 } & Model & -34.98 & -17.35 & -3.37 & 3.61 & 17.54 & 35.00 \\
\hline
\end{tabular}


This model is also verified against PSCAD benchmark for three different $L_{\text {arm }}$ are shown in Table V. It can be seen that the matching between the two models is excellent.

TABLE V. TEST RESULTS FOR DIFFERENT $\mathrm{L}_{\mathrm{ARM}}$ WITH CCSC (P=1PU)

\begin{tabular}{|c|c|c|c|c|}
\hline \multicolumn{2}{|c|}{$\mathrm{P}=1 \mathrm{pu}$} & $\mathrm{L}_{\mathrm{arm}}=0.02 \mathrm{H}$ & $\mathrm{L}_{\text {arm }}=0.04 \mathrm{H}$ & $\mathrm{L}_{\text {arm }}=0.06 \mathrm{H}$ \\
\hline \multirow{2}{*}{$I_{\text {diffo }}(K A)$} & PSCAD & 0.518 & 0.518 & 0.518 \\
\cline { 2 - 5 } & Model & 0.518 & 0.518 & 0.518 \\
\hline \multirow{2}{*}{$e_{d}(K V)$} & PSCAD & 208.5 & 208.5 & 208.5 \\
\cline { 2 - 5 } & Model & 208.5 & 208.5 & 208.5 \\
\hline \multirow{2}{*}{$e_{q}(K V)$} & PSCAD & -20.00 & -25.00 & -30.00 \\
\cline { 2 - 5 } & Model & -19.95 & -24.97 & -29.98 \\
\hline
\end{tabular}

\section{MODEL APPLICATION EXAMPLE AND COMPARISON WITH} 2-LEVEL VSC

This section considers a simple AC system with MMC as shown in Fig 2, and calculates converter AC voltages for a range of control inputs. Appendix III shows how the MMC model is analytically connected to the AC system model in order to determine current vector components.

In the MMC model in (37) it is seen that the last term $0.5 M V_{d c}$ is the well-known 2-level VSC converter equation. It is interesting to examine if MMC can be modelled as a 2-level VSC, by neglecting the load dependent first terms in (37). Therefore a comparable 2-level VSC converter model which has series inductance as MMC total inductance (transformer inductance and half arm inductance, $\left.L_{T_{-} V S C}=L_{T_{-} M M C}+L_{a r m} / 2\right)$ is also tested. The test system is shown in Fig. 2 where the converter is either MMC or VSC. The $M_{q}$ varies from -0.07 to +0.07 while $M_{d}$ is kept equal to 0.92 .

Fig. 4 shows the $\mathrm{AC}$ side voltages for the two converters. It is seen that MMC and 2-level VSC have different responses and therefore load dependent terms in MMC model have
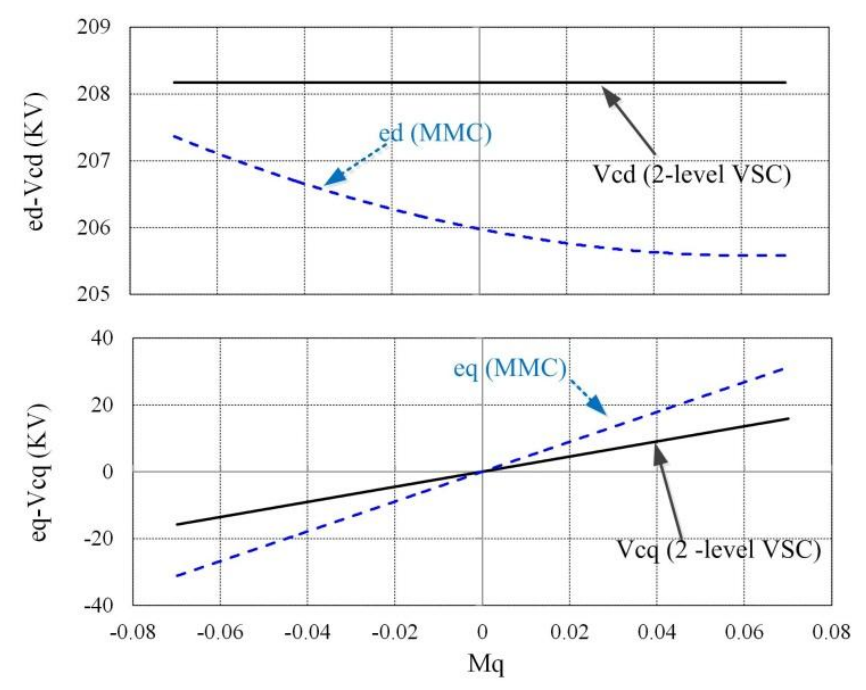

Fig. 4. Comparison of AC equivalent voltages for MMC and VSC

significant influence. It is concluded that an MMC should not be represented using simple 2-level VSC models. For the same control signal $M_{q}$, the load dependent terms in MMC act to increase the converter voltage $e_{q}$. The MMC therefore responds as a controllable voltage source with a series capacitor.
This can also be confirmed analytically. If the converter resistance is neglected in (37) $R_{\text {arm }}=0$, than the MMC model has the following format:

$$
\begin{aligned}
& e_{d}=-\frac{I_{V q}}{\omega C_{M M C}}+\frac{M_{d} V_{d c}}{2} \\
& e_{d}=\frac{I_{V d}}{\omega C_{M M C}}+\frac{M_{q} V_{d c}}{2}
\end{aligned}
$$

Where the equivalent MMC capacitance is:

$$
C_{M M C}=64 C^{a r m} / 8-3 M_{d}^{2}+M_{q}^{2}
$$

assuming further that in most operating conditions: $M_{d}^{2}+M_{q}^{2} \approx 1$, eq. (40) becomes:

$$
C_{M M C}=64 C^{a r m} / 5
$$

The model in (39) implies that MMC responds like a 2-level VSC with an additional series capacitance $C_{M M C}$. Fig 5 shows the equivalent simplified MMC model.

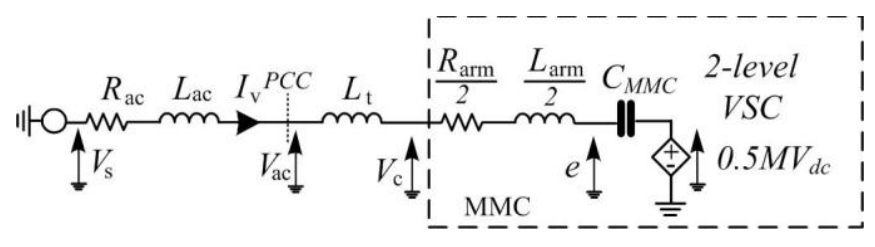

Fig. 5. Simplified MMC model connected to the AC grid.

\section{CONCLUSION}

A detailed phasor model for MMC is proposed considering variables in the $d q$ frame rotating at fundamental frequency and the coordinate frame $d 2 q 2$ rotating at double the fundamental frequency. A set of generic equations for manipulating nonlinear terms directly in $d q$ frame is first developed. It is concluded that there is significant coupling between the zero sequence variables, variables in $d q$ frame and second harmonic variables.

The circulating current control is also modelled and it is found that very small magnitudes of second harmonic control signals can completely cancel the circulating current second harmonics. It is proposed to neglect the second harmonic control signals and this enabled derivation of a 2-equation final MMC model. If converter losses are also neglected, it is demonstrated that MMC behaves as 2-level VSC with an equivalent series capacitance which depends on the control signal magnitudes.

The validity and accuracy of the proposed phasor models is verified against a benchmark model in PSCAD. The models are tested for different power flows and various $L_{\text {arm }}$. The results for all cases verify accuracy of the proposed phasor models.

\section{APPENDIX I. DQ FRAME ALGEBRA}

Eq. (11) and others require multiplication of two phasor signals in $d q$ frame. Here, a general formula for signal multiplication in $d q$ frame is derived. Starting with a time domain expression for two signals $X(t)$ and $Y(t)$, each 
consisting of zero sequence, fundamental and second harmonic:

$$
\begin{aligned}
& X(t)=X_{0}+X_{D} \cos \omega t+X_{Q} \sin \omega t+X_{D 2} \cos 2 \omega t+X_{Q 2} \sin 2 \omega t \\
& Y(t)=Y_{0}+Y_{D} \cos \omega t+Y_{Q} \sin \omega t+Y_{D 2} \cos 2 \omega t+Y_{Q 2} \sin 2 \omega t
\end{aligned}
$$

The product signal $Z(t)=X(t) \times Y(t)$ will also contain zero sequence, basic frequency, second harmonic but also third and fourth harmonics:

$$
\begin{aligned}
& Z(t)=\underbrace{\left(X_{0} Y_{0}+\frac{X_{\mathrm{D}} Y_{\mathrm{D}}}{2}+\frac{X_{\mathrm{Q}} Y_{\mathrm{Q}}}{2}+\frac{X_{D 2} Y_{D 2}}{2}+\frac{X_{Q 2} Y_{Q 2}}{2}\right)}_{Z_{0}}+ \\
& \underbrace{\left(X_{\mathrm{D}} Y_{0}+X_{0} Y_{D}+\frac{1}{2} X_{D 2} Y_{D}+\frac{1}{2} X_{Q 2} Y_{Q}+\frac{1}{2} Y_{D 2} X_{D}+\frac{1}{2} Y_{Q 2} X_{Q}\right)}_{Z_{d}} \cos \omega t \\
& +\underbrace{\left(X_{\mathrm{Q}} Y_{0}+X_{0} Y_{Q}-\frac{1}{2} X_{D 2} Y_{Q}+\frac{1}{2} X_{Q 2} Y_{D}-\frac{1}{2} Y_{D 2} X_{Q}+\frac{1}{2} Y_{Q 2} X_{D}\right)}_{Z_{q}} \sin \omega t \\
& +\underbrace{\left(\frac{X_{\mathrm{D}} Y_{\mathrm{D}}}{2}-\frac{X_{\mathrm{Q}} Y_{\mathrm{Q}}}{2}+X_{D 2} Y_{0}+Y_{D 2} X_{0}\right)}_{Z_{d 2}} \cos (\omega t) \\
& +\underbrace{\left(\frac{X_{\mathrm{Q}} Y_{\mathrm{D}}}{2}+\frac{X_{\mathrm{D}} Y_{\mathrm{Q}}}{2}+X_{Q 2} Y_{0}+Y_{Q 2} X_{0}\right)}_{Z_{q 2}} \sin (2 \omega t)
\end{aligned}
$$

where third and fourth harmonics are neglected. The corresponding terms in $d q$ frame are indicated in (43).

It is also required to derive the $d q$ components of differential signals as shown on left hand side of (1). A general oscillating signal $x(t)$ is differentiated in $d q$ frame as:

$$
\begin{aligned}
& \frac{d}{d t} x(t)=\frac{d}{d t}\left[X_{0}+X_{d} \cos \omega t-X_{q} \sin \omega t\right] \\
& \frac{d}{d t} x(t)=-\omega X_{d} \sin \omega t-\omega X_{q} \cos \omega t
\end{aligned}
$$

Therefore, in the rotating frame differential of signal $x(t)$ is:

$$
\left(\frac{d}{d t} x(t)\right)_{d q}=-k \omega X_{Q_{d}}+k \omega X_{D_{q}}
$$

Where $k=1$ for basic frequency and $k=2$ for second harmonic.

APPENDIX II. DERIVATION OF $I_{D I F F}$ AND RESONANT $L_{A R M}$ By substituting Eq. (12) and (16) in (20) and rearranging:

$$
\begin{aligned}
& B \quad A I_{d i f f d 2}+B I_{\text {diffq } 2}=C \\
& -B \quad A I_{d i f f q_{2}}-B I_{\text {diffd } 2}=D
\end{aligned}
$$

where

$$
\begin{aligned}
& A=8 \omega^{2} L_{\text {arm }} C^{a r m}-\frac{2+m^{2}}{4}, \quad m^{2}=M_{d}^{2}+M_{q}^{2} \\
& B=4 \omega C^{a r m} R_{a r m} \\
& C=\frac{M_{d}^{2}-M_{q}^{2}}{2} I_{\text {diff } 0}-\frac{3 M_{d} I_{V d}}{8}+\frac{3 M_{q} I_{V q}}{8} \\
& D=M_{d} M_{q} I_{\text {diff } 0}-\frac{3 M_{d} I_{V q}}{8}-\frac{3 M_{q} I_{V d}}{8}
\end{aligned}
$$

From (46) differential currents are determined:

$$
\begin{aligned}
& I_{\text {diffd } 2}=A C-B D / A^{2}+B^{2} \\
& I_{d i f f q_{2}}=A D+B C / A^{2}+B^{2}
\end{aligned}
$$

The magnitude of $I_{d i f f 2}$ from (48) becomes:

$$
I_{\text {diff } 2}=\sqrt{C^{2}+D^{2} / A^{2}+B^{2}}, \quad A^{2}+B^{2} \neq 0
$$

By differentiating $I_{\text {diff2 }}$ with respect to $L_{a r m}$ and considering that $C$ and $D$ do not depend on $L_{a r m}$,

$$
\frac{\partial I_{d i f f 2}}{\partial L_{a r m}}=\frac{\partial I_{d i f f 2}}{\partial A} \frac{\partial A}{\partial L_{a r m}}=\frac{-C^{2}+D^{2}{ }^{1 / 2} A}{A^{2}+B^{2}} 8 \omega^{2 / 2} C^{a r m}=0
$$

Eq. (50) requires that $A=0$. Note that if $B=0$, the final condition is also $A=0$. Therefore,

$$
8 \omega^{2} L_{\text {arm }} C^{\text {arm }}-\frac{2+m^{2}}{4}=0 \Rightarrow L_{\text {arm_res }}=\frac{2+m^{2}}{32 \omega^{2} C^{a r m}}
$$

APPENDIX III. DERIVATION OF AC CURRENT IN AN AC SYSTEM WITH MMC

The ac side KVL equation is:

$$
\begin{gathered}
I_{V d}+j I_{V q} \quad R_{x}+j X_{x}=V_{a c}-e_{d}-j e_{q} \\
\left\{\begin{array}{c}
R_{x} I_{V d}-X_{x} I_{V q}=V_{a c}-e_{d} \\
R_{x} I_{V q}+X_{x} I_{V d}=-e_{q}
\end{array}\right.
\end{gathered}
$$

where $R_{x}=R_{\text {arm }} / 2$ and $X_{x}=\left(L_{t}+L_{\text {arm }} / 2\right) \omega$.

By substituting (37) in (52) and rearranging:

$$
\begin{gathered}
I_{V d}=\frac{R_{x} K_{22}+X_{x} K_{12}}{K_{11} K_{22}-K_{12} K_{21}} V_{a c}+\frac{-K_{31} K_{22}-K_{32} K_{12}}{K_{11} K_{22}-K_{12} K_{21}} V_{D C} \\
I_{V q}=\frac{R_{x} K_{21}+X_{x} K_{11}}{K_{12} K_{21}-K_{11} K_{22}} V_{a c}+\frac{-K_{31} K_{21}-K_{32} K_{11}}{K_{12} K_{21}-K_{11} K_{22}} V_{D C}
\end{gathered}
$$

where

$$
\begin{aligned}
& K_{11}=R_{x}^{2}+X_{x}^{2}+R_{x} A_{11}+X_{x} A_{21} \\
& K_{12}=R_{x} A_{12}+X_{x} A_{22} \\
& K_{31}=R_{x} b_{1}+X_{x} b_{2} \\
& K_{21}=-X_{x} A_{11}+R_{x} A_{21} \\
& K_{22}=R_{x}^{2}+X_{x}^{2}-X_{x} A_{12}+R_{x} A_{22} \\
& K_{32}=X_{x} b_{1}-R_{x} b_{2}
\end{aligned}
$$

The ac side equation from $V_{S}$ to $V_{a c}$ is

$$
\left\{\begin{array}{c}
R_{a c} I_{V d}-X_{a c} I_{V q}+V_{a c}=V_{s d} \\
X_{a c} I_{V d}+R_{a c} I_{V q}=V_{s q}
\end{array}\right.
$$

which can be written in following form

$$
R_{a c} I_{V d}-X_{a c} I_{V q}+V_{a c}{ }^{2}+X_{a c} I_{V d}+R_{a c} I_{V q}{ }^{2}=V_{S}^{2}
$$

By substituting (46) in (49),

$$
R^{2}+T^{2} V_{a c}^{2}+2 Q R+2 S T V_{a c}+Q^{2}+S^{2}-V_{S}^{2}=0
$$


where

$$
\begin{aligned}
& Q=\frac{-K_{31} K_{22}-K_{32} K_{12}}{K_{11} K_{22}-K_{12} K_{21}} R_{a c} V_{D C}+\frac{K_{31} K_{21} K_{32} K_{11}}{K_{12} K_{21}-K_{11} K_{22}} X_{a c} V_{D C} \\
& R=\left(\frac{R_{x} K_{22}+X_{x} K_{12}}{K_{11} K_{22}-K_{12} K_{21}} R_{a c}-\frac{R_{x} K_{21}+X_{x} K_{11}}{K_{12} K_{21}-K_{11} K_{22}} X_{a c}+1\right) \\
& S=\frac{-K_{31} K_{22}-K_{32} K_{12}}{K_{11} K_{22}-K_{12} K_{21}} X_{a c} V_{D C}+\frac{-K_{31} K_{21}-K_{32} K_{11}}{K_{12} K_{21}-K_{11} K_{22}} R_{a c} V_{D C} \\
& T=\left(\frac{R_{x} K_{22}+X_{x} K_{12}}{K_{11} K_{22}-K_{12} K_{21}} X_{a c}+\frac{R_{x} K_{21}+X_{x} K_{11}}{K_{12} K_{21}-K_{11} K_{22}} R_{a c}\right)
\end{aligned}
$$

By calculating $V_{a c}$ from (57), $I_{V d}$ and $I_{V q}$ can be obtained from (53).

\section{REFERENCES}

[1] A. Antonopoulos, L. Angquist, and H. Nee, "On Dynamics and Voltage Control of the Modular Multilevel Converter," Proc. Power Electronics and Applications (EPE), Barcelona, Spain, Sep. 2009, p. 10

[2] .M. Hagiwara, and H. Akagi, "Control and Experiment of PulsewidthModulated Modular Multilevel Converters" IEEE Transactions on Power Electronics, vol.24, no.7, pp.1737-1746, July 2009

[3] S. Allebrod, R. Hamerski, and R. Marquardt, "New transformerless, scalable Modular Multilevel Converters for HVDC-transmission," Power Electronics Specialists Conference, 2008. PESC 2008. IEEE, pp.174,179, 15-19 June 2008

[4] P.S. Jones, and C. C. Davidson, "Calculation of power losses for MMCbased VSC HVDC stations," 15th European Conference on Power Electronics and Applications (EPE), 2013, pp.1,10, 2-6 Sept. 2013

[5] U.N. Gnanarathna, A.M. Gole, and R.P. Jayasinghe, "Efficient Modeling of Modular Multilevel HVDC Converters (MMC) on Electromagnetic Transient Simulation Programs", IEEE Transactions on Power Delivery, vol.26, no.1, pp.316-324, Jan. 2011

[6] J. Peralta, H. Saad, S. Dennetiere, J. Mahseredjian, and S. Nguefeu, "Detailed and Averaged Models for a 401-Level MMC-HVDC System," IEEE Transactions on Power Delivery, vol.27, no.3, 2012 pp.1501-08.

[7] H. Saad, J. Peralta, S. Dennetiere, and J. Mahseredjian, "Dynamic Averaged and Simplified Models for MMC-Based HVDC Transmission Systems", IEEE transactions on Power delivery, vol. 28, pp. 1723-1730, July 2013.

[8] Sawata R. Deore, Pranav B. Darji and Anil M. Kulkarni, "Dynamic Phasor Modeling of Modular Multi-level Converters", 7th IEEE International Conference on Industrial and Information Systems (ICIIS), Aug. 2012.

[9] J.Qin and M. Saeedfard "Predictive Control of Modular Multilevel Converter for a Back-to-back HVDC System" IEEE Transactions on Power delivery, Vol 27, issue 3, 2012, pp. 1538-1547.

[10] T.Soong and P.W.Lehn, "Internal Power flow of a Modular Multilevel Converter with Distributed Energy Resources" IEEE journal of Emerging and Selected Topics in Power Electronics, Vol 2, issue 4, 2014, pp 1127-1138.

[11] Ilves, K., Antonopoulos, A., Norrga, S., Nee, H.-P. "Steady-state analysis of interaction between harmonic components of arm and line quantities of modular multilevel converters" IEEE Transactions on Power Electronics, 27 (1), 2012, pp. 57-68

[12] Wang, C., Hao, Q., Ooi, B.-T. "Reduction of low-frequency harmonics in modular multilevel converters (MMCs) by harmonic function analysis" IET Generation, Transmission and Distribution, 8 (2), 2014 pp. 328-338

[13] Q. Tu, Z. Xu, and L. Xu, "Reduced switching-frequency modulation and circulating current suppression for modular multilevel converters," IEEE Transactions on Power Delivery, vol. 23, no. 3, pp. 2009-17, Jul. 2011.

[14] Tu, Q., Xu, Z., Zhang, J. "Circulating current suppressing controller in modular multilevel converter", IECON Proceedings (Industrial Electronics Conference), art. no. 5675048, 2010, pp. 3198-3202

\section{BIOGRAPHIES}

Dragan Jovcic (SM'06, M'00, S'97) obtained a Diploma Engineer degree in Control Engineering from the University of Belgrade, Serbia in 1993 and a $\mathrm{Ph} . \mathrm{D}$. degree in Electrical Engineering from the University of Auckland, New Zealand in 1999. He is currently a professor with the University of Aberdeen, Scotland where he has been since 2004. In 2008 he held visiting professor post at McGill University, Montreal, Canada. He also worked as a lecturer with University of Ulster, in the period 2000-2004 and as a design Engineer in the New Zealand power industry, Wellington, in the period 1999-2000. His research interests lie in the HVDC, FACTS, DC grids and control systems.

Ali akbar Jamshidifar obtained his B.Sc., M.Sc., and Ph.D.degrees in Control Engineering from Sharif University of Technology, Iran University of Science and Technology, and Amirkabir University of Technology in 1992, 1996, and 2008 respectively. He has been as a researcher with Iranian Research Organization for Science and Technology (IROST) since 1999. He is currently a research fellow with the University of Aberdeen, Scotland. His research interests include the modeling and control of HVDC. 